\title{
Clifford systems, Clifford structures, and their canonical differential forms
}

\author{
Kai Brynne M. Boydon ${ }^{1} \cdot$ Paolo Piccinni ${ }^{2}$
}

Received: 28 August 2020 / Accepted: 19 November 2020 / Published online: 8 December 2020

(c) The Author(s) 2020

\begin{abstract}
A comparison among different constructions in $\mathbb{W}^{2} \cong \mathbb{R}^{8}$ of the quaternionic 4-form $\Phi_{\mathrm{Sp}(2) \operatorname{Sp}(1)}$ and of the Cayley calibration $\Phi_{\text {Spin(7) }}$ shows that one can start for them from the same collections of "Kähler 2-forms", entering both in quaternion Kähler and in Spin(7) geometry. This comparison relates with the notions of even Clifford structure and of Clifford system. Going to dimension 16, similar constructions allow to write explicit formulas in $\mathbb{R}^{16}$ for the canonical 4-forms $\Phi_{\mathrm{Spin}(8)}$ and $\Phi_{\mathrm{Spin}(7) \mathrm{U}(1)}$, associated with Clifford systems related with the subgroups $\operatorname{Spin}(8)$ and $\operatorname{Spin}(7) \mathrm{U}(1)$ of $\mathrm{SO}(16)$. We characterize the calibrated 4-planes of the 4-forms $\Phi_{\mathrm{Spin}(8)}$ and $\Phi_{\mathrm{Spin}(7) \mathrm{U}(1)}$, extending in two different ways the notion of Cayley 4-plane to dimension 16.
\end{abstract}

Keywords Octonions · Clifford system $\cdot$ Clifford structure $\cdot$ Calibration $\cdot$ Canonical form

Mathematics Subject Classification Primary 53C26 - 53C27 · 53C38

\section{Introduction}

In 1989 R. Bryant and R. Harvey defined the following calibration, of interest in hyperkähler geometry [6]:

$$
\Phi_{K}=-\frac{1}{2} \omega_{R_{i}}^{2}-\frac{1}{2} \omega_{R_{j}}^{2}+\frac{1}{2} \omega_{R_{k}}^{2} \in \Lambda^{4} \mathbb{W}^{n}
$$

Communicated by Vicente Cortés.

Paolo Piccinni

piccinni@mat.uniroma1.it

Kai Brynne M. Boydon

kbboydon@math.upd.edu.ph

1 Institute of Mathematics, University of the Philippines Diliman, Quezon City, Philippines

2 Dipartimento di Matematica, Sapienza Università di Roma, Piazzale Aldo Moro 2, 00185 Roma, Italy 
In this definition, $\left(\omega_{R_{i}}, \omega_{R_{j}}, \omega_{R_{k}}\right)$ are the Kähler 2-forms of the hypercomplex structure $\left(R_{i}, R_{j}, R_{k}\right)$, defined by multiplications on the right by unit quaternions $(i, j, k)$ on the space $\mathbb{R}^{4 n} \cong \mathbb{H}^{n}$.

When $n=2$, the Bryant-Harvey calibration $\Phi_{K}$ relates with Spin(7) geometry. This is easily recognized by using the map

$$
L: \mathbb{T}^{2} \rightarrow \mathbb{O}, \quad L\left(h_{1}, h_{2}\right)=h_{1}+\left(k h_{2} \bar{k}\right) e \in \mathbb{O},
$$

from pairs of quaternions to octonions, that yields the identity

$$
L^{*} \Phi_{\operatorname{Spin}(7)}=\Phi_{K}
$$

Here $\Phi_{\text {Spin(7) }} \in \Lambda^{4} \mathbb{R}^{8}$ is the Spin(7) 4-form, or Cayley calibration, studied since the R. Harvey and H. B. Lawson's foundational paper [11], and defined through the scalar product and the double cross product of $\mathbb{R}^{8} \cong \mathbb{O}$ :

$$
\Phi_{\operatorname{Spin}(7)}(x, y, z, w)=<x, y \times z \times w>=<x, y(\bar{z} w)>,
$$

assuming here orthogonal $y, z, w \in \mathbb{O}$.

The present paper collects some of the results in the first author Ph.D. thesis [3], inspired from viewing formula (1.1) as a way of constructing the Cayley calibration $\Phi_{\text {Spin(7) }}$ through the 2-forms $\omega_{R_{i}}, \omega_{R_{j}}, \omega_{R_{k}}$. As well known, by summing the squares of the latter 2-forms one gets another remarkable calibration, namely the quaternionic right 4-form $\Omega_{R}$. Thus $\omega_{R_{i}}, \omega_{R_{j}}, \omega_{R_{k}}$, somehow building blocks for quaternionic geometry, enter also in $\operatorname{Spin}(7)$ geometry.

A first result is the following Theorem 1.1, a kind of "other way around" of formula (1.1). To state it, recall that the Cayley calibration $\Phi_{\text {Spin(7) }}$ can also be constructed as sum of squares of "Kähler 2-forms" associated with complex structures on $\mathbb{R}^{8} \cong \mathbb{O}$, defined by the unit octonions. In fact, cf. [19, Prop.10]:

$$
\Phi_{\operatorname{Spin}(7)}=-\frac{1}{6}\left(\phi_{i}^{2}+\phi_{j}^{2}+\cdots+\phi_{h}^{2}\right)=\frac{1}{6}\left(\varphi_{i j}^{2}+\phi_{i k}^{2}+\varphi_{i k}^{2}+\cdots+\varphi_{g h}^{2}\right) .
$$

Here $\phi_{i}, \phi_{j}, \ldots, \phi_{h}$ are the Kähler 2-forms associated with the 7 complex structures $R_{i}, R_{j}, \ldots, R_{h}$ on $\mathbb{R}^{8} \cong \mathbb{O}$, the right multiplications by the unit octonions $i, j, k, e, f, g, h$, and $\phi_{i j}, \phi_{i k}, \ldots, \phi_{g h}$ are the Kähler 2-forms associated with the 21 complex structures $R_{i j}=R_{i} \circ R_{j}, R_{i k}=R_{i} \circ R_{k}, \ldots, R_{g h}=R_{g} \circ R_{h}$, compositions of them.

Theorem 1.1 The right quaternionic 4-form $\Omega_{R} \in \Lambda^{4} \mathbb{D}^{2}$ can be obtained from the Kähler forms $\phi_{i}, \phi_{j}, \ldots, \phi_{h}$ associated with the complex structures $R_{i}, R_{j}, \ldots, R_{h}$ as:

$$
\Omega_{R}=2\left[\phi_{i}^{2}+\phi_{j}^{2}+\phi_{k}^{2}-\phi_{e}^{2}-\phi_{f}^{2}-\phi_{g}^{2}-\phi_{h}^{2}\right]
$$

Moreover, by selecting any five out of the seven $\left(J_{1}=R_{i}, J_{2}=R_{j}, \ldots, J_{7}=R_{h}\right)$ and by looking at the matrix $\zeta=\left(\zeta_{\alpha \beta}\right) \in \mathfrak{g} \mathfrak{o}(5)$ of Kähler 2-forms of their compositions $J_{\alpha \beta}=J_{\alpha} \circ J_{\beta}$, one can get the left quaternionic 4-form $\Omega_{L}$ as

$$
\Omega_{L}=-\frac{1}{2} \sum_{\alpha<\beta} \zeta_{\alpha \beta}^{2},
$$

up to a permutation or change of signs of some coordinates in $\mathbb{R}^{8}$. 
On the same direction as in Bryant-Harvey's formula (1.1), a similar result is the following (cf. Sect. 4 for more details):

Theorem 1.2 The Cayley calibration $\Phi_{\text {Spin(7) }} \in \Lambda^{4} \mathbb{R}^{8}$ can be obtained from the Kähler 2-forms $\eta_{\alpha \beta}(1 \leq \alpha<\beta \leq 5)$ associated to complex structures $\mathcal{I}_{\alpha \beta}^{L}=\mathcal{I}_{\alpha}^{L} \circ \mathcal{I}_{\beta}^{L}$, where $\mathcal{I}_{1}^{L}, \ldots \mathcal{I}_{5}^{L}$ are anti-commuting self-dual involutions in $\mathbb{R}^{8}$. Namely:

$$
\Phi_{\operatorname{Spin}(7)}=\frac{1}{4}\left[\eta_{12}^{2}+\eta_{13}^{2}+\eta_{24}^{2}+\eta_{34}^{2}-\eta_{23}^{2}-\eta_{14}^{2}-\eta_{15}^{2}-\eta_{25}^{2}-\eta_{35}^{2}-\eta_{45}^{2}\right],
$$

and on the other hand one can get the right quaternion Kähler $\Omega_{R}$ as:

$$
\Omega_{R}=-\frac{1}{2}\left[\eta_{12}^{2}+\eta_{13}^{2}+\eta_{24}^{2}+\eta_{34}^{2}+\eta_{23}^{2}+\eta_{14}^{2}+\eta_{15}^{2}+\eta_{25}^{2}+\eta_{35}^{2}+\eta_{45}^{2}\right]
$$

Moving to dimension 16 and in Sect. 6, we consider two exterior 4-forms

$$
\Phi_{\mathrm{Spin}(8)}, \quad \Phi_{\mathrm{Spin}(7) \mathrm{U}(1)} \in \Lambda^{4} \mathbb{R}^{16},
$$

canonically associated with subgroups $\operatorname{Spin}(8), \operatorname{Spin}(7) \mathrm{U}(1) \subset \mathrm{SO}(16)$, and we write their explicit expressions in the 16 coordinates. We will see in the next two statements which 4-planes of $\mathbb{R}^{16}$ are calibrated by $\Phi_{\mathrm{Spin}(8)}$ and by $\Phi_{\mathrm{Spin}(7) \mathrm{U}(1)}$.

The 4-forms $\Phi_{\text {Spin(8) }}$, $\Phi_{\text {Spin(7)U(1) }}$ and the respective calibrated 4-planes can be compared with other calibrations in $\mathbb{R}^{16}$, in particular with the previously mentioned Bryant-Harvey 4-form $\Phi_{K}$. It is thus appropriate to remind the main theorem in [6, Theorem 2.27], namely that, in any $\mathbb{U}^{n} \cong \mathbb{R}^{4 n}$, the Bryant-Harvey 4-form $\Phi_{K}$ calibrates the Cayley 4-planes that are contained in a quaternionic 2-dimensional vector subspace $W_{\mathbb{H}}^{2} \subset \mathbb{H}^{n}$.

Here we prove:

Theorem 1.3 The oriented 4-planes of $\mathbb{R}^{16} \cong \mathbb{O}^{2}$ calibrated by the 4-form $\Phi_{\mathrm{Spin}(8)}$ are the transversal Cayley 4-planes, i.e. the 4-planes $P$ such that both projections $\pi(P), \pi^{\prime}(P)$ on the two summands in $\mathbb{O}^{2}=\mathbb{O} \oplus \mathbb{O}^{\prime}$ are two dimensional and both invariant by a same complex structure $u \in S^{6} \subset \operatorname{Im} \mathbb{O}$.

Also, by recalling that $\mathbb{O}^{2}$ decomposes in the union of octonionic lines

$$
\ell_{m}=\{(x, m x), x \in \mathbb{O}, m \in \mathbb{O} \cup \infty\},
$$

meeting pairwise only at $(0,0) \in \mathbb{O}^{2}$, we can state:

Theorem 1.4 The oriented 4-planes of $\mathbb{R}^{16}$ calibrated by the 4-form $\Phi_{\mathrm{Spin}(7) \mathrm{U}(1)}$ are the ones that are invariant under a complex structure $u \in S^{6} \subset \operatorname{Im} \mathbb{D}$ and that are contained in an octonionic line $\ell_{m} \subset \mathbb{O} \oplus \mathbb{O}$, where only $m \in \mathbb{R}$ and $m=\infty$ are allowed. Thus they are "Cayley 4-planes", contained in the oriented 8-planes that are the mentioned octonionic lines with $m \in \mathbb{R} \cup \infty$.

\section{Preliminaries}

The multiplication in the algebra $\mathbb{O}$ of octonions can be defined from the one in quaternions $\mathbb{W}$ through the Cayley-Dickson process: if $x=h_{1}+h_{2} e, x^{\prime}=h_{1}^{\prime}+h_{2}^{\prime} e \in \mathbb{O}$, then 


$$
x x^{\prime}=\left(h_{1} h_{1}^{\prime}-\bar{h}_{2}^{\prime} h_{2}\right)+\left(h_{2} \bar{h}_{1}^{\prime}+h_{2}^{\prime} h_{1}\right) e,
$$

where product of quaternions is used on the right hand side and $\bar{h}_{1}^{\prime}, \bar{h}_{2}^{\prime}$ are the conjugates of $h_{1}^{\prime}, h_{2}^{\prime} \in \mathbb{H}$. Like for quaternions, the conjugation $\bar{x}=\bar{h}_{1}-h_{2} e$ in $\mathbb{O}$ relates with the noncommutativity: $\overline{x x^{\prime}}=\bar{x}^{\prime} \bar{x}$. One has also the associator $\left[x, x^{\prime}, x^{\prime \prime}\right]=\left(x x^{\prime}\right) x^{\prime \prime}-x\left(x^{\prime} x^{\prime \prime}\right)$, that vanishes whenever two among $x, x^{\prime}, x^{\prime \prime} \in \mathbb{O}$ are equal or conjugate.

The identification $x=h_{1}+h_{2} e \in \mathbb{O} \leftrightarrow\left(h_{1}, h_{2}\right) \in \mathbb{M}^{2}$, used in the previous formula, is not an isomorphism of (left or right) quaternionic vector spaces. To get an isomorphism one has instead to go through the following hypercomplex structure $(I, J, K)$ on $\mathbb{R}^{8} \cong \mathbb{O}$. For $x=h_{1}+h_{2} e \in \mathbb{O}$, where $\left(h_{1}, h_{2}\right) \in \mathbb{R}^{2}$, define

$$
I(x)=x \cdot i, J(x)=x \cdot j, K(x)=(x \cdot i) \cdot j
$$

or equivalently

$$
\left.I\left(h_{1}, h_{2}\right)=\left(h_{1} i,-h_{2} i\right), J\left(h_{1}, h_{2}\right)\right)=\left(h_{1} j,-h_{2} j\right), K(x)=\left(h_{1} k, h_{2} k\right) .
$$

This observation goes likely back to the very discovery of octonions in the mid-1800s. The alternative approach to the same isomorphism used in our Introduction does not seem however to have appeared before 1989, when R. Bryant and R. Harvey [6] looked at the map

$$
L: \mathbb{T}^{2} \rightarrow \mathbb{O}, \quad L\left(h_{1}, h_{2}\right)=h_{1}+\left(k h_{2} \bar{k}\right) e \in \mathbb{O},
$$

and observed it satisfies

$$
L\left[\left(h_{1}, h_{2}\right) i\right]=h_{1} i+\left(k h_{2} i \bar{k}\right) e, \quad L\left[\left(h_{1}, h_{2}\right) j\right]=h_{1} j+\left(k h_{2} j \bar{k}\right) e, L\left[\left(h_{1}, h_{2}\right) k\right]=h_{1} k+\left(k h_{2}\right) e .
$$

This, in terms of $x_{1}=h_{1}, x_{2}=k h_{2} \bar{k}$ and of the octonion $x=x_{1}+x_{2} e$, can be read exactly as in our previous approach:

$$
L\left[\left(h_{1}, h_{2}\right) i\right]=x \cdot i, L\left[\left(h_{1}, h_{2}\right) j\right]=x \cdot j, L\left[\left(h_{1}, h_{2}\right) k\right]=(x \cdot i) \cdot j,
$$

and as mentioned $L^{*} \Phi_{\operatorname{Spin}(7)}=\Phi_{K}$.

\section{The quaternionic 4-form and the Cayley calibration in $\mathbb{R}^{8}$}

A possible way to produce 4-forms canonically associated with some $G$-structures is through the notion of Clifford system. We recall the definition, originally given in the context of isoparametric hypersurfaces, cf. [9].

Definition 3.1 A Clifford system on a Riemannian manifold $(M, g)$ is a vector sub-bundle $E^{r} \subset$ End $T M$ locally spanned by self-adjoint anti-commuting involutions $\mathcal{I}_{1}, \ldots, \mathcal{I}_{r}$. Thus $I_{\alpha}^{2}=\mathrm{Id}, \quad \mathcal{I}_{\alpha}^{*}=\mathcal{I}_{\alpha}, \quad \mathcal{I}_{\alpha} \circ \mathcal{I}_{\beta}=-\mathcal{I}_{\beta} \circ \mathcal{I}_{\alpha}$, and the $\mathcal{I}_{\alpha}$ are required to be related, in the intersections of trivializing sets, by matrices of $\mathrm{SO}(r)$. The rank $r$ of $E$ is said to be the rank of the Clifford system.

Possible ranks of irreducible Clifford systems on $\mathbb{R}^{N}$ are classified, up to $N=32$, in Table 1. 
In particular, the Clifford system of rank 3 in $\mathbb{R}^{4}$ can be defined by the classical Pauli matrices:

$$
\mathcal{I}_{1}=\left(\begin{array}{ll}
0 & 1 \\
1 & 0
\end{array}\right), \mathcal{I}_{2}=\left(\begin{array}{cc}
0 & -i \\
i & 0
\end{array}\right), \mathcal{I}_{3}=\left(\begin{array}{cc}
1 & 0 \\
0 & -1
\end{array}\right) \in \mathrm{U}(2) \subset \mathrm{SO}(4),
$$

and the Clifford system of rank 5 in $\mathbb{R}^{8}$ by the following similar (right) quaternionic Pauli matrices:

$$
\begin{aligned}
& \mathcal{I}_{1}=\left(\begin{array}{cc}
0 & \mathrm{Id} \\
\mathrm{Id} & 0
\end{array}\right), \mathcal{I}_{2}=\left(\begin{array}{cc}
0 & -R_{i} \\
R_{i} & 0
\end{array}\right), \mathcal{I}_{3}=\left(\begin{array}{cc}
0 & -R_{j} \\
R_{j} & 0
\end{array}\right), \\
& \mathcal{I}_{4}=\left(\begin{array}{cc}
0 & -R_{k} \\
R_{k} & 0
\end{array}\right), \mathcal{I}_{5}=\left(\begin{array}{cc}
\mathrm{Id} & 0 \\
0 & -\mathrm{Id}
\end{array}\right) \in \mathrm{Sp}(2) \subset \mathrm{SO}(8),
\end{aligned}
$$

where as before $R_{i}, R_{j}, R_{k}$ denote the multiplication on the right by $i, j, k$ on $\mathbb{U}^{2} \cong \mathbb{R}^{8}$.

According to Table 1 , there is also a Clifford system with $r=4$ in $\mathbb{R}^{8}$, explicitly defined by selecting e.g.

$$
\mathcal{I}_{1}=\left(\begin{array}{cc}
0 & \mathrm{Id} \\
\mathrm{Id} & 0
\end{array}\right), \mathcal{I}_{2}=\left(\begin{array}{cc}
0 & -R_{i} \\
R_{i} & 0
\end{array}\right), \mathcal{I}_{3}=\left(\begin{array}{cc}
0 & -R_{j} \\
R_{j} & 0
\end{array}\right), \mathcal{I}_{4}=\left(\begin{array}{cc}
0 & -R_{k} \\
R_{k} & 0
\end{array}\right) \text {. }
$$

Going back to rank $r=5$, from the quaternionic Pauli matrices $\mathcal{I}_{1}, \mathcal{I}_{2}, \mathcal{I}_{3}, \mathcal{I}_{4}, \mathcal{I}_{5}$, one gets the 10 complex structures on $\mathbb{R}^{8}$

$$
\mathcal{I}_{\alpha \beta}=\mathcal{I}_{\alpha} \circ \mathcal{I}_{\beta} \quad \text { for } \quad 1 \leq \alpha<\beta \leq 5 .
$$

Their Kähler forms $\theta_{\alpha \beta}$ give rise to a $5 \times 5$ skew-symmetric matrix

$$
\theta_{R}=\left(\theta_{\alpha \beta}\right),
$$

and one can easily see that both the following matrices of Kähler 2-forms

$$
\theta_{R}=\left(\theta_{\alpha \beta}\right) \in \mathfrak{s} \mathfrak{o}(5) \quad \text { and } \quad \omega_{L}=\left(\begin{array}{ccc}
0 & \omega_{L_{i}} & \omega_{L_{j}} \\
-\omega_{L_{i}} & 0 & \omega_{L_{k}} \\
-\omega_{L_{j}} & -\omega_{L_{k}} & 0
\end{array}\right) \in \mathfrak{g} \mathfrak{o}(3)
$$

allow to write the (left) quaternionic 4-form of $\llbracket^{2}$ as

$$
\Omega_{L}=-\frac{1}{2} \sum_{\alpha<\beta} \theta_{\alpha \beta}^{2}=\left[\omega_{L_{i}}^{2}+\omega_{L_{j}}^{2}+\omega_{L_{k}}^{2}\right] .
$$

On the other hand, as mentioned in the Introduction, the subgroup $\operatorname{Spin}(7) \subset \mathrm{SO}(8)$ (generated by the right translation $R_{u}, u \in S^{6} \subset \operatorname{Im} \mathbb{O}$ ) gives rise to the Cayley calibration $\Phi_{\text {Spin(7) }} \in \Lambda^{4}$ :

Table 1 Rank of irreducible Clifford systems in $\mathbb{R}^{N}$

\begin{tabular}{lllllllllllll}
\hline Dimension $N$ & 2 & 4 & 8 & 8 & 16 & 16 & 16 & 16 & 32 & 64 & 64 & $\ldots$ \\
Rank $r$ & 2 & 3 & 4 & 5 & 6 & 7 & 8 & 9 & 10 & 11 & 12 & $\ldots$ \\
\hline
\end{tabular}




$$
\Phi_{\operatorname{Spin}(7)}=-\frac{1}{6}\left[\phi_{i}^{2}+\phi_{j}^{2}+\ldots \phi_{h}^{2}\right]=\frac{1}{6} \sum_{\alpha<\beta} \zeta_{\alpha \beta}^{2}
$$

Here $\phi_{i}, \phi_{j}, \phi_{k}, \phi_{e}, \phi_{f}, \phi_{g}, \phi_{h}$. are the Kähler 2-forms associated with the complex structures $\left(J_{1}, J_{2}, J_{3}, J_{4}, J_{5}, J_{6}, J_{7}\right)=\left(R_{i}, R_{j}, R_{k}, R_{e}, R_{f}, R_{g}, R_{h}\right)$, and $\zeta=\left(\zeta_{\alpha \beta}\right) \in \mathfrak{g} \mathfrak{o}$ (7) is the matrix of the Kähler 2-forms of compositions $J_{\alpha \beta}=J_{\alpha} \circ J_{\beta}$.

It is worth to recall that under the action of $\operatorname{Sp}(2) \operatorname{Sp}(1)$, the space of exterior 2-forms $\Lambda^{2} \mathbb{R}^{8}$ decomposes as

$$
\Lambda^{2}=\Lambda_{10}^{2} \oplus \Lambda_{15}^{2} \oplus \Lambda_{3}^{2},
$$

(cf. [22, page 125] as well as [23, page 93]). Here lower indices denote the dimensions of irreducible components. Here $\Lambda_{10}^{2} \cong \mathfrak{g p}(2)$ is generated by the Kähler forms $\theta_{\alpha \beta}$ of the $J_{\alpha \beta}(\alpha<\beta)$, compositions of the five quaternionic Pauli matrices, and $\Lambda_{3}^{2} \cong \mathfrak{g p}(1)$ is generated by the Kähler forms $\omega_{L_{i}}, \omega_{L_{j}}, \omega_{L_{k}}$.

By denoting by $\tau_{2}$ the second coefficient in the characteristic polynomial of the involved skew-symmetric matrices, we can rewrite formula (3.2) of $\Omega_{L}$ as:

$$
\Omega_{L}=-\frac{1}{2} \tau_{2}\left(\theta_{R}\right)=\tau_{2}\left(\omega_{L}\right),
$$

where $\theta_{R}=\left(\theta_{\alpha \beta}\right) \in \mathfrak{g} \mathfrak{o}(5)$, and $\omega_{L}=\left(\begin{array}{ccc}0 & \omega_{L_{i}} & \omega_{L_{j}} \\ -\omega_{L_{i}} & 0 & \omega_{L_{k}} \\ -\omega_{L_{j}} & -\omega_{L_{k}} & 0\end{array}\right) \in \mathfrak{g} \mathfrak{p}(3)$.

Similarly, under the $\operatorname{Spin}(7)$ action one gets the decomposition:

$$
\Lambda^{2}=\Lambda_{7}^{2} \oplus \Lambda_{21}^{2},
$$

see [13, page 256] or [14, page 47]. Here $\Lambda_{7}^{2}$ is generated by the Kähler forms $\phi_{\alpha}$ of the $J_{\alpha}=R_{i}, R_{j}, \ldots, R_{h}$ and $\Lambda_{21}^{2} \cong \mathfrak{g p i n}(7)$ is generated by the Kähler forms $\zeta_{\alpha \beta}$ of the $J_{\alpha} \circ J_{\beta}(\alpha<\beta)$. Thus, in the $\tau_{2}$ notation:

$$
\Phi_{\operatorname{Spin}(7)}=-\frac{1}{6} \sum \phi_{\alpha}^{2}=\frac{1}{6} \tau_{2}(\zeta), \quad \zeta=\left(\zeta_{\alpha \beta}\right) \in \mathfrak{g} \mathfrak{o}(7)
$$

All the exterior 4-forms $\theta_{\alpha \beta}, \phi_{\alpha}$ and the $\zeta_{\alpha \beta}$ have been studied systematically as calibrations in the space $\mathbb{R}^{8}$, cf. [8].

\section{Proof of Theorems 1.1 and 1.2}

The matrix $\eta=\left(\eta_{\alpha \beta}\right) \in \mathfrak{g} \mathfrak{o}(5)$ in the statement of Theorem 1.2 is defined as follows. Let $\mathcal{I}_{\alpha}^{L}(\alpha=1, \ldots, 5)$ be the left quaternionic Pauli matrices defined as in (3.1) but by using the left quaternionic multiplications $L_{i}, L_{j}, L_{k}$ by $i, j . k$. If $\mathcal{I}_{\alpha \beta}^{L}=\mathcal{I}_{\alpha}^{L} \circ \mathcal{I}_{\beta}^{L}$ and if $\eta_{\alpha \beta}$ are the Kähler 2-forms associated to $\mathcal{I}_{\alpha \beta}^{L}$, a computation shows that

$$
2 \Omega_{R}=\eta_{12}^{2}+\eta_{13}^{2}+\eta_{24}^{2}+\eta_{34}^{2}+\eta_{23}^{2}+\eta_{14}^{2}+\eta_{15}^{2}+\eta_{25}^{2}+\eta_{35}^{2}+\eta_{45}^{2},
$$

and note the symmetry with the first identity in formula (3.2).

We express now the 2 -forms $\theta_{\alpha \beta}$ and $\eta_{\alpha \beta}$ in the coordinates of $\mathbb{R}^{8}$, using the following abridged notations. Let $\left\{d x_{1}, \ldots, d x_{8}\right\} \subset \Lambda^{1} \mathbb{R}^{8}$ be the standard basis of 1 -forms in $\mathbb{R}^{8}$. Then 
$\alpha \beta$ (scriptsize) denotes $d x_{\alpha} \wedge d x_{\beta}$ and $\alpha \beta \gamma \delta$ denotes $d x_{\alpha} \wedge d x_{\beta} \wedge d x_{\gamma} \wedge d x_{\delta}$, and $\star$ denotes the Hodge star, so that $a+\star=a+\star a$. One gets:

$$
\begin{aligned}
& \theta_{12}=-12+34+56-78, \\
& \theta_{13}=-13-24+57+68, \\
& \theta_{14}=-14+23+58-67, \\
& \theta_{23}=-14+23-58+67, \\
& \theta_{24}=+13+24+57+68, \\
& \theta_{34}=-12+34-56+78,
\end{aligned}
$$

and

$$
\begin{aligned}
& \theta_{15}=-15-26-37-48, \\
& \theta_{25}=-16+25+38-47, \\
& \theta_{35}=-17-28+35+46, \\
& \theta_{45}=-18+27-36+45,
\end{aligned}
$$

so that, if $\theta=\left(\theta_{\alpha \beta}\right)$,

$$
\begin{aligned}
\tau_{2}(\theta) & =\theta_{12}^{2}+\theta_{13}^{2}+\cdots+\theta_{45}^{2} \\
& =-121234-41256-41357+41368-41278-41467-41458+\star=-2 \Omega_{L} .
\end{aligned}
$$

Next:

$$
\begin{aligned}
& \eta_{12}=-12-34+56+78, \\
& \eta_{13}=-13+24+57-68, \\
& \eta_{14}=-14-23+58+67, \\
& \eta_{23}=+14+23+58+67, \\
& \eta_{24}=-13+24-57+68, \\
& \eta_{34}=+12+34+56+78, \\
& \eta_{15}=-15-26-37-48, \\
& \eta_{25}=-16+25-38+47, \\
& \eta_{35}=-17+28+35-46, \\
& \eta_{45}=-18-27+36+45,
\end{aligned}
$$

and, if $\eta=\left(\eta_{\alpha \beta}\right)$,

$$
\begin{aligned}
\tau_{2}(\eta) & =\eta_{12}^{2}+\eta_{13}^{2}+\cdots+\eta_{45}^{2} \\
& =121234-41256-41357-41368+41278+41467-41458+\star=-2 \Omega_{R} .
\end{aligned}
$$

Similarly: 


$$
\begin{aligned}
& \phi_{i}=-12+34+56-78, \\
& \phi_{j}=-13-24+57+68, \\
& \phi_{k}=-14+23+58-67, \\
& \phi_{e}=-15-26-37-48, \\
& \phi_{f}=-16+25-38+47, \\
& \phi_{g}=-17+28+35-46, \\
& \phi_{h}=-18-27+36+45,
\end{aligned}
$$

and one easily deduces also formulas for the $\zeta_{\alpha \beta}$ (cf. $\left.[3,19]\right)$. Then, by (1.2):

$$
\Phi_{\text {Spin(7) }}=1234+1256+1357+1368-1278-1467+1458+\star .
$$

By computing the squares of the 2-forms in (4.6) (4.4) and comparing with Formulas (4.5), (4.3), (4.7), the identities listed in Theorems 1.1 and 1.2 are recognized.

\section{Even Clifford structures in dimension 8}

We recall first the following notion, proposed in 2011 by A. Moroianu and U. Semmelmann, [17].

Definition 5.1 Let $(M, g)$ be a Riemannian manifold. An even Clifford structure is the choice of an oriented Euclidean vector bundle $E^{r}$ of rank $r \geq 2$ over $M$, together with a bundle morphism $\varphi$ from the even Clifford algebra bundle

$$
\varphi: \mathrm{Cl}^{\text {even }} E^{r} \rightarrow \operatorname{End} T M \quad \text { such that } \Lambda^{2} E^{r} \hookrightarrow \mathrm{End}^{-} T M .
$$

$r$ is called the rank of the even Clifford structure.

The even Clifford structure $E$ is said to be parallel if there exists a metric connection $\nabla^{E}$ on $E$ such that $\varphi$ is connection preserving, i.e.

$$
\varphi\left(\nabla_{X}^{E} \sigma\right)=\nabla_{X}^{g} \varphi(\sigma)
$$

for every tangent vector $X \in T M$ and section $\sigma$ of $\mathrm{Cl}^{\text {even }} E$, where $\nabla^{g}$ is the Levi Civita connection.

Rank 2, 3, 4 parallel even Clifford structures are equivalent to complex Kähler, quaternion Kähler, product of two quaternion Kähler. Besides them, higher rank parallel non-flat even Clifford structures in dimension 8 are listed in Table 2, cf. [17].

A class of examples of even Clifford structures are those coming from Clifford systems as defined in Sect. 3. Namely, if the vector sub-bundle $E^{r} \subset$ End $T M$, locally spanned by self-adjoint anticommuting involutions $\mathcal{I}_{1}, \ldots, \mathcal{I}_{r}$, defines the Clifford system, then one easily recognizes that through the compositions $\mathcal{I}_{\alpha \beta}=\mathcal{I}_{\alpha} \circ \mathcal{I}_{\beta}$, the Clifford morphism $\varphi: \mathrm{Cl}^{\text {even }}\left(E^{r}\right) \rightarrow$ End $T M$ is well defined.

An example is given by the first row of Table 2, where the quaternion Kähler structure is constructed via the local $\mathcal{I}_{\alpha \beta}=\mathcal{I}_{\alpha} \circ \mathcal{I}_{\beta}$ defined as in Sect. 3, by using on the model space $\mathbb{\mathbb { Z } ^ { 2 }}$ the quaternionic Pauli matrices. The remaining three rows of Table 2 correspond to 
Table 2 Parallel non-flat even Clifford structures of rank $\geq 5$ in $M^{8}$

\begin{tabular}{ll}
\hline$r$ & $M$ \\
\hline 5 & Quaternion Kähler \\
6 & Kähler \\
7 & Spin(7) holonomy \\
8 & Riemannian \\
\hline
\end{tabular}

Table 3 Generators and associated 4-forms

\begin{tabular}{llll}
\hline $\mathrm{r}$ & $M$ & Clifford bundle generators & Associated 4-form \\
\hline 5 & $\mathrm{qK}$ & $\mathcal{I}_{1}, \mathcal{I}_{2}, \mathcal{I}_{3}, \mathcal{I}_{4}, \mathcal{I}_{5}$ & $\Omega_{L}=-\frac{1}{2} \tau_{2}\left(\theta_{\alpha \beta}\right), \Omega_{R}=-\frac{1}{2} \tau_{2}\left(\eta_{\alpha \beta}\right)$ \\
6 & Kähler & $J_{1}, J_{2}, J_{3}, J_{4}, J_{5}, J_{6}$ & $\Phi_{\operatorname{Spin}(6)}=\tau_{2}\left(\zeta_{\alpha \beta}\right)=-5 \omega^{2}$ \\
7 & Spin(7) hol & $J_{1}, J_{2}, J_{3}, J_{4}, J_{5}, J_{6}, J_{7}$ & $\Phi_{\operatorname{Spin}(7)}=-\frac{1}{6} \sum \phi_{\alpha}^{2}=\frac{1}{6} \tau_{2}\left(\zeta_{\alpha \beta}\right)$ \\
8 & Riemannian & $I, J_{1}, J_{2}, J_{3}, J_{4}, J_{5}, J_{6}, J_{7}$ & $\Phi_{\mathrm{SO}(8)}=\tau_{2}\left(\psi_{\alpha \beta}\right)=0$ \\
\hline
\end{tabular}

essential even Clifford structures, i. e. to even Clifford structures that cannot be defined throw a Clifford system, cf. [21] for a discussion on this notion.

Table 3 gives a description of the Clifford bundle generators and of the canonically associated 4-form for each of the four even Clifford structures on $\mathbb{R}^{8}$.

Here $\mathcal{I}_{1}, \mathcal{I}_{2}, \mathcal{I}_{3}, \mathcal{I}_{4}, \mathcal{I}_{5}$ are the (left or right) quaternionic Pauli matrices, $\left(\theta_{\alpha \beta}\right),\left(\eta_{\alpha \beta}\right)$ are like in Sect. 4. Notations $\left(J_{1}, J_{2}, J_{3}, J_{4}, J_{5}, J_{6}, J_{7}\right)=\left(R_{i}, R_{j}, R_{k}, R_{e}, R_{f}, R_{g}, R_{h}\right)$ are also used, $\phi_{\alpha}$ is the Kähler form of $J_{\alpha}$ and $\zeta_{\alpha \beta}$ is the Kähler form of $J_{\alpha} \circ J_{\beta}$. Finally, $\left(\psi_{\alpha \beta}\right) \in \mathfrak{g} \mathfrak{o}(8)$, with entries $\pm \phi_{\alpha}$ in the first line and column and with entries $\zeta_{\alpha \beta} \in \mathfrak{g} \mathfrak{o}(7)$.

It is of course desirable to give examples of Riemannian manifolds $\left(M^{8}, g\right)$ supporting both a $S p(2) \cdot S p(1)$ and a $S p i n(7)$ structure. Rarely the metric $g$ can be the same for both structures, but this is possible of course for parallelizable $\left(M^{8}, g\right)$. On this respect, homogeneous $\left(M^{8}, g\right)$ with an invariant $\operatorname{Spin}(7)$ structure have been recently classified [1], by making use of the following topological condition for compact oriented spin $M^{8}$ :

$$
p_{1}^{2}(M)-4 p_{2}(M)+8 \chi(M)=0 .
$$

Some of the obtained examples are parallelizable, e. g. diffeomorphic to $S^{7} \times S^{1}$ and $S^{5} \times S^{3}$. On the latter, $S^{5} \times S^{3}$, using two natural parallelizations, one can define two $\operatorname{Spin}(7)$ structures, both of general type (in the 1986 M. Fernández Spin(7) framework), and the hyperhermitian structure associated with one of them corresponds to a family of CalabiEckmann [20].

To get examples of 8-dimensional manifolds that admit both a locally conformally hyperkähler metric $g$ and a locally conformal parallel Spin(7) metric, that is either the same $g$ as before, or a different metric $g^{\prime}$, a good point to start with is the class of compact 3-Sasakian 7-dimensional manifolds $\left(\mathcal{S}^{7}, g\right)$. Many examples of such $\left(\mathcal{S}^{7}, g\right)$ and with arbitrary second Betti numbers have been given by Ch. Boyer, K. Galicki et al, cf. [5]. In particular, recall that given the 3-Sasakian $\left(\mathcal{S}^{7}, g\right)$ one gets a locally conformally hyperkähler metric $g$ on the product $\mathcal{S}^{7} \times S^{1}[18]$. This can also be expressed by saying that the 3 -Sasakian metric $g$ has the property of being nearly parallel $\mathrm{G}_{2}$, and in particular with 3 linearly independent Killing spinors, cf. [4, pp. 536-538]. Moreover the differentiable manifold $\mathcal{S}^{7}$ 
admits, besides the 3-Sasakian metric $g$, another metric $g^{\prime}$ that is also nearly parallel $\mathrm{G}_{2}$ but proper, i. e. with only one non zero Killing spinor. This allows to extend the metrics $g$ and $g^{\prime}$ to the product with $S^{1}$ and to get both the properties of locally conformally hyperkähler and locally conformally parallel $\operatorname{Spin}(7)$ on $\left(M^{8}, g\right)=\left(\mathcal{S}^{7} \times S^{1}, g\right)$ and of locally conformally parallel $\operatorname{Spin}(7)$ on $\left(M^{8}, g^{\prime}\right)=\left(\mathcal{S}^{7} \times S^{1}, g^{\prime}\right)$, cf. also [12].

Further examples of 8-dimensional differentiable manifolds admitting both a $\mathrm{Sp}(2) \cdot \mathrm{Sp}(1)$-structure with respect to a metric $g$ and a $\operatorname{Spin}(7)$-structure with respect to a metric $g^{\prime}$ include the Wolf spaces $\mathbb{H} P^{2}$ and $\mathrm{G}_{2} / \mathrm{SO}(4)$, cf. [1]. Finally, the non singular sextic $Y=\left\{\left[z_{0}, \ldots, z_{5}\right] \in \mathbb{C} P^{5}, z_{0}^{6}+\cdots+z_{5}^{6}=0\right\}$ is also an example, where a metric $g$ giving an almost quaternionic structure is insured by a result in [7], and a metric $g^{\prime}$ with holonomy $\mathrm{SU}(4) \subset \operatorname{Spin}(7)$ by Calabi-Yau theorem, cf. [13, p. 139].

\section{Dimension 16}

A Clifford system with $r=9$ in $\mathbb{O}^{2} \cong \mathbb{R}^{16}$ is given by the following octonionic Pauli matrices:

$$
\begin{aligned}
& \mathcal{I}_{1}=\left(\begin{array}{cc}
0 & \mathrm{Id} \\
\mathrm{Id} & 0
\end{array}\right), \mathcal{I}_{2}=\left(\begin{array}{cc}
0 & -R_{i} \\
R_{i} & 0
\end{array}\right), \mathcal{I}_{3}=\left(\begin{array}{cc}
0 & -R_{j} \\
R_{j} & 0
\end{array}\right), \\
& \mathcal{I}_{4}=\left(\begin{array}{cc}
0 & -R_{k} \\
R_{k} & 0
\end{array}\right), \mathcal{I}_{5}=\left(\begin{array}{cc}
0 & -R_{e} \\
R_{e} & 0
\end{array}\right), \mathcal{I}_{6}=\left(\begin{array}{cc}
0 & -R_{f} \\
R_{f} & 0
\end{array}\right), \\
& \mathcal{I}_{7}=\left(\begin{array}{cc}
0 & -R_{g} \\
R_{g} & 0
\end{array}\right), \mathcal{I}_{8}=\left(\begin{array}{cc}
0 & -R_{h} \\
R_{h} & 0
\end{array}\right), \mathcal{I}_{9}=\left(\begin{array}{cc}
\mathrm{Id} & 0 \\
0 & -\mathrm{Id}
\end{array}\right) \in \mathrm{SO}(16),
\end{aligned}
$$

and of course now $R_{i}, R_{j}, \ldots, R_{h}$ denote the multiplication on the right by the unit octonions $i, j, \ldots, h$ on $\mathbb{O}^{2} \cong \mathbb{R}^{16}$.

Looking back at Table 1 , we see that in $\mathbb{R}^{16}$ there are also irreducible Clifford systems with $r=8,7,6$. According to [21], convenient choices are the following:

$$
r=8: \mathcal{I}_{1}, \ldots, \mathcal{I}_{8}, \quad r=7: \mathcal{I}_{2}, \ldots, \mathcal{I}_{8}, \quad r=6: \mathcal{I}_{1}, \mathcal{I}_{2}, \mathcal{I}_{3}, \mathcal{I}_{4}, \mathcal{I}_{5}, \mathcal{I}_{9} .
$$

It is now worth to remind the following parallel situations in complex, quaternionic and octonionic geometry. The groups $\mathrm{U}(2) \subset \mathrm{SO}(4), \mathrm{Sp}(2) \cdot \mathrm{Sp}(1) \subset \mathrm{SO}(8), \mathrm{Spin}(9) \subset \mathrm{SO}(16)$ are the stabilizers of the vector subspaces

$$
E^{3} \subset \operatorname{End}^{+}\left(\mathbb{R}^{4}\right), \quad E^{5} \subset \operatorname{End}^{+}\left(\mathbb{R}^{8}\right), \quad E^{9} \subset \operatorname{End}^{+}\left(\mathbb{R}^{16}\right)
$$

spanned respectively by the Pauli, quaternionic Pauli, octonionic Pauli matrices.

Moreover, $\mathrm{U}(2), \mathrm{Sp}(2) \cdot \mathrm{Sp}(1), \operatorname{Spin}(9)$ are symmetry groups of the Hopf fibrations respectively:

$$
S^{3} \stackrel{S^{1}}{\longrightarrow} S^{2} \cong \mathbb{C} P^{1}, \quad S^{7} \stackrel{S^{3}}{\longrightarrow} S^{4} \cong \mathbb{H} P^{1}, \quad S^{15} \stackrel{S^{7}}{\longrightarrow} S^{8} \cong \mathbb{O} P^{1} .
$$

Finally, $U(2), \operatorname{Sp}(2) \cdot \operatorname{Sp}(1), \operatorname{Spin}(9)$ are stabilizers in $\Lambda^{2} \mathbb{C}^{2}, \Lambda^{4} \mathbb{M}^{2}, \Lambda^{8} \mathbb{O}^{2}$ of the following canonically associated forms, cf. [2]:

$$
\Phi_{\mathrm{U}(2)}=\int_{\mathbb{C} P^{1}} p_{\ell}^{*} v_{\ell} d \ell \in \Lambda^{2}, \quad \Phi_{\mathrm{Sp}(2) \cdot \mathrm{sp}(1)}=\int_{\mathbb{H} P^{1}} p_{\ell}^{*} v_{\ell} d \ell \in \Lambda^{4}, \quad \Phi_{\mathrm{Spin}(9)}=\int_{\mathbb{O P 1}} p_{\ell}^{*} v_{\ell} d \ell \in \Lambda^{8},
$$


where $v_{\ell}$ is the volume form on the line $\ell \stackrel{\text { def }}{=}\{(x, m x)\}$ or $\ell \stackrel{\text { def }}{=}\{(0, y)\}$ in $\mathbb{C}^{2}$ or $\mathbb{U}^{2}$ or $\mathbb{O}^{2}$,

$$
p_{\ell}: \mathbb{C}^{2} \cong \mathbb{R}^{4} \text { or } \mathbb{H}^{2} \cong \mathbb{R}^{8} \text { or } \mathbb{O}^{2} \cong \mathbb{R}^{16} \longrightarrow \ell
$$

is the projection on the line $\ell$ ), and note that the integral formula is based on the volume of distinguished planes. In the three cases one gets in this way the Kähler 2-form of $\mathbb{C}^{2}$, the quaternion Kähler 4-form of $\mathbb{\boxplus}^{2}$ and the canonical 8-form of $\mathbb{O}^{2}$.

\section{Rank 8, 7 and 6 Clifford systems on $\mathbb{R}^{16}$}

Look now closer at the nine octonionic Pauli matrices, that define a rank 9 Clifford system in $\mathbb{R}^{16}$, and at the choices among them that give rise to ranks $r=8,7,6$ (cf. previous Section). The compositions $\mathcal{I}_{\alpha \beta}=\mathcal{I}_{\alpha} \circ \mathcal{I}_{\beta}, \alpha<\beta$, for all choices $r=6,7,8,9$ are bases of the Lie algebras

$$
\mathfrak{G p i n}(6) \subset \mathfrak{g p i n}(7) \subset \mathfrak{g p i n}(8) \subset \mathfrak{g p i n}(9) \subset \mathfrak{g} \mathfrak{o}(16)
$$

Like in the previous Sections, we can write the matrices of Kähler forms $\psi_{\alpha \beta}$ associated to $\mathcal{I}_{\alpha \beta}$, and we use for them the following notations:

$$
\psi^{A}=\left(\psi_{\alpha \beta}\right) \in \mathfrak{g} \mathfrak{o}(6), \quad \psi^{B}=\left(\psi_{\alpha \beta}\right) \in \mathfrak{g} \mathfrak{o}(7), \quad \psi^{C}=\left(\psi_{\alpha \beta}\right) \in \mathfrak{g} \mathfrak{o}(8), \quad \psi^{D}=\left(\psi_{\alpha \beta}\right) \in \mathfrak{g} \mathfrak{o}(9) .
$$

The second coefficients $\tau_{2}$ of their characteristic polynomial give rise to the following invariant 4-forms

$$
\tau_{2}\left(\psi^{A}\right), \quad \tau_{2}\left(\psi^{B}\right), \quad \tau_{2}\left(\psi^{C}\right), \quad \tau_{2}\left(\psi^{D}\right) \in \Lambda^{4} \mathbb{R}^{16}
$$

that can be written in (the differentials of) the coordinates of $\mathbb{R}^{16}=\mathbb{O} \oplus \mathbb{O}$ :

$$
1,2,3,4,5,6,7,8 ; 1^{\prime}, 2^{\prime}, 3^{\prime}, 4^{\prime}, 5^{\prime}, 6^{\prime}, 7^{\prime}, 8^{\prime} \text {. }
$$

We recall in particular that in the $\operatorname{Spin}(9)$ situation, the following identity holds:

$$
\tau_{2}\left(\psi^{D}\right)=0
$$

and this gives evidence to the next coefficient $\tau_{4}\left(\psi^{D}\right) \in \Lambda^{8}$, proportional to the 8 -form $\Phi_{\text {Spin(9) }}$, as studied in [19].

\section{The 4-forms $\Phi_{\operatorname{Spin}(8)}$ and $\Phi_{\operatorname{Spin}(7) U(1)}$}

Look now only at $\mathcal{I}_{1}, \ldots, \mathcal{I}_{8}$ and at the matrix

$$
\psi^{C}=\left(\psi_{\alpha \beta}\right) \in \mathfrak{g} \mathfrak{o}(8)
$$

of Kähler forms associated to $\mathcal{I}_{\alpha \beta}=\mathcal{I}_{\alpha} \circ \mathcal{I}_{\beta}$. By using coordinate 1-forms

$$
1,2,3,4,5,6,7,8 ; 1^{\prime}, 2^{\prime}, 3^{\prime}, 4^{\prime}, 5^{\prime}, 6^{\prime}, 7^{\prime}, 8^{\prime},
$$

an explicit computation, based on the formulas in [19] for the $\psi_{\alpha \beta}$, yields: 


$$
\begin{aligned}
& \Phi_{\operatorname{Spin}(8)}=\frac{1}{4} \tau_{2}\left(\psi^{C}\right)=\frac{1}{4} \sum_{1=\alpha<\beta}^{8} \psi_{\alpha \beta}^{2}=\mathbf{1 2 3 4}+\mathbf{1 2 5 6}-\mathbf{1 2 7 8}+\mathbf{1 3 5 7}+\mathbf{1 3 6 8}+\mathbf{1 4 5 8} \\
& -\mathbf{1 4 6 7}-\mathbf{2 3 5 8}+\mathbf{2 3 6 7}+\mathbf{2 4 5 7}+\mathbf{2 4 6 8}-\mathbf{3 4 5 6}+\mathbf{3 4 7 8}+\mathbf{5 6 7 8}+\sum_{1=a<b}^{8} a b a^{\prime} b^{\prime} \in \Lambda^{4} \mathbb{R}^{16} \text {. }
\end{aligned}
$$

Here boldface notations have the following meaning:

$$
a b c d=a b c^{\prime} d^{\prime}-a b^{\prime} c d^{\prime}+a b^{\prime} c^{\prime} d+a^{\prime} b c d^{\prime}-a^{\prime} b c^{\prime} d+a^{\prime} b^{\prime} c d .
$$

By excluding now the Kähler forms involving $\mathcal{I}_{1}$ and $\mathcal{I}_{9}$, one gets the matrix $\psi^{B}=\left(\psi_{\alpha \beta}\right) \in \mathfrak{g} \mathfrak{o}(7)$. Now similar computations lead to:

$$
\begin{aligned}
\Phi_{\mathrm{Spin}(7) \mathrm{U}(1)=} & \tau_{2}\left(\psi^{B}\right)=6[1234+1256 \\
& -1278+1357+1368+1458-1467-2358+2367+2457+2468-3456+3478+5678] \\
& +6\left[1^{\prime} 2^{\prime} 3^{\prime} 4^{\prime}+1^{\prime} 2^{\prime} 5^{\prime} 6^{\prime}-1^{\prime} 2^{\prime} 7^{\prime} 8^{\prime}+1^{\prime} 3^{\prime} 5^{\prime} 7^{\prime}+1^{\prime} 3^{\prime} 6^{\prime} 8^{\prime}+1^{\prime} 4^{\prime} 5^{\prime} 8^{\prime}-1^{\prime} 4^{\prime} 6^{\prime} 7^{\prime}-2^{\prime} 3^{\prime} 5^{\prime} 8^{\prime}\right. \\
& \left.+2^{\prime} 3^{\prime} 6^{\prime} 7^{\prime}+2^{\prime} 4^{\prime} 5^{\prime} 7^{\prime}+2^{\prime} 4^{\prime} 6^{\prime} 8^{\prime}-3^{\prime} 4^{\prime} 5^{\prime} 6^{\prime}+3^{\prime} 4^{\prime} 7^{\prime} 8^{\prime}+5^{\prime} 6^{\prime} 7^{\prime} 8\right]+6 \sum_{1=a<b}^{8} a b a^{\prime} b^{\prime} \\
& +2[\mathbf{1 2 3 4}+\mathbf{1 2 5 6}-\mathbf{1 2 7 8}+\mathbf{1 3 5 7}+\mathbf{1 3 6 8}+\mathbf{1 4 5 8}-\mathbf{1 4 6 7}-\mathbf{2 3 5 8} \\
& +\mathbf{2 3 6 7}+\mathbf{2 4 5 7}+\mathbf{2 4 6 8}-\mathbf{3 4 5 6}+\mathbf{3 4 7 8}+\mathbf{5 6 7 8}] \in \Lambda^{4} \mathbb{R}^{16},
\end{aligned}
$$

where boldface notations have the same meaning as before. The presence of the factor U(1) in the group $\operatorname{Spin}(7) \mathrm{U}(1)$ is here due to a computation showing that matrices in $\mathrm{SO}(16)$ commuting with the seven involutions $\mathcal{I}_{2}, \ldots \mathcal{I}_{8}$ are a $U(1)$ subgroup, well identified in [3, Chapter 6, p. 44] (cf. also proof of Theorem 1.4 below).

We take this opportunity to remark that the expression of $\Phi_{\text {Spin(7)U(1) }}$ written in the paper [21] contains some errors in the coefficients as well as some missing terms, and has to be corrected by the present one. Note also that $\Phi_{\mathrm{Spin}(7) \mathrm{U}(1)}$ restricts, on any of the two summands of $\mathbb{R}^{16}=\mathbb{R}^{8} \oplus \mathbb{R}^{8}$, and up to a factor 6 , to the usual Cayley calibration of [11].

We are now ready for the proofs of Theorems 1.3 and 1.4. The following notion has already implicitly introduced in the statement of Theorem 1.3.

Definition 8.1 Let $P$ be a 4-plane in the real vector space $\mathbb{R}^{16} \cong \mathbb{O}^{2}=\mathbb{O} \oplus \mathbb{O}^{\prime}$, and let $\pi: \mathbb{O}^{2} \rightarrow \mathbb{O}$ and $\pi^{\prime}: \mathbb{O}^{2} \rightarrow \mathbb{O}^{\prime}$ be the orthogonal projections to $\mathbb{O}$ and $\mathbb{O}^{\prime} . P$ is said to be a transversal Cayley 4-plane if both its projections $\pi(P), \pi^{\prime}(P)$ are 2-dimensional and invariant under a same complex structure $u \in S^{6} \subset \operatorname{Im} \mathbb{O}$.

Proof of Theorem 1.3 Recall that Spin(8) can be characterized as the subgroup of the following matrices $A \in \mathrm{SO}(16)$ :

$$
A=\left(\begin{array}{ll}
a_{+} & 0 \\
0 & a_{-}
\end{array}\right)
$$

where $a_{+}, a_{-} \in \mathrm{SO}(8)$ are triality companions, i. e. and for any $v \in \mathbb{O}$ there exists a $w \in \mathbb{O}$ such that $R_{w}=a_{+} R_{v} a_{-}^{t}$ (cf. [10, p. 278-279]). It follows that $\operatorname{Spin}(8)$ contains the diagonal $\operatorname{Spin}(7)_{\Delta}$ (characterized by choices $a_{+}=a_{-}$) and acts transitively on transversal 4-planes of $\mathbb{R}^{16}$. On the other hand the 4-form $\Phi_{\operatorname{Spin}(8)}$ is invariant under the action of $\operatorname{Spin}(8)$. Thus, since $\Phi_{\text {Spin }(8)}$ takes value 1 on the 4-plane spanned by the coordinates $121^{\prime} 2^{\prime}, \Phi_{\text {Spin }(8)}$ takes value 1 on any tranversal Cayley 4-plane in $\mathbb{R}^{16}$. 
Next, let $Q$ be any 4-plane of $\mathbb{R}^{16}$. By looking at the expression of $\Phi_{\mathrm{Spin}(8)}$, we see that the only possibilities for having non zero value on $Q$ are that $\pi(Q)$ and $\pi^{\prime}(Q)$ are 2-dimensional. For such 4-planes $Q$ we can use the following canonical form with respect to the complex structure $i \in S^{6}$ :

$$
Q=\left[e_{1} \wedge\left(R_{i} e_{1} \cos \theta+e_{2} \sin \theta\right)\right] \oplus\left[e_{1}^{\prime} \wedge\left(R_{i} e_{1}^{\prime} \cos \theta^{\prime}+e_{2}^{\prime} \sin \theta^{\prime}\right)\right]
$$

where the pairs $e_{1}, e_{2}$ and $e_{1}^{\prime}, e_{2}^{\prime}$ are both orthonormal and respectively in $\mathbb{O}$ and in $\mathbb{O}^{\prime}$, and with angles limited by $0 \leq \theta \leq \frac{\pi}{2}$ and $\theta \leq \theta^{\prime} \leq \pi-\theta$. The above canonical form for $Q$ is a small variation of the canonical forms that are used in a proof of the classical Wirtinger's inequaliy (cf. [16, p.6]) and in characterizations of Cayley 4-planes in $\mathbb{R}^{8}$ in the HarveyLawson foundational paper (cf. [11, p. 121]). Its proof follows the steps of proof of the mentioned canonical form, as explained in details in [16]. From this canonical form we see that $\Phi_{\operatorname{Spin}(8)}(Q) \leq 1$ for any 4-plane $Q$, and that the equality holds only if $\theta=\theta^{\prime}=0$, i. e. for transversal Cayley 4-planes.

Proof of Theorem 1.4 The leading terms in the expression of $\Phi_{\mathrm{Spin}(7) \mathrm{U}(1)}$ are those with coefficient 6 , thus terms involving only coordinates among 12345678 , or only coordinates among $1^{\prime} 2^{\prime} 3^{\prime} 4^{\prime} 5^{\prime} 6^{\prime} 7^{\prime} 8^{\prime}$, or terms $a b a^{\prime} b^{\prime}$. Look first at the first and second types of terms. We already mentioned that the restriction of $\Phi_{\operatorname{Spin}(7) \mathrm{U}(1)}$ to any of the summands in $\mathbb{O}^{2}=\mathbb{O} \oplus \mathbb{O}^{\prime}$ is the usual Cayley calibration in $\mathbb{R}^{8}$, whose calibrated 4-planes are the Cayley planes. Thus, for the first two types of terms, we get as calibrated 4-planes just the Cayley 4-planes that are contained in the octonionic lines with slope $m=0$ and $m=\infty$. In the remaining case of terms $a b a^{\prime} b^{\prime}$ one gets as calibrated 4-planes the transversal Cayley 4-planes that are contained in the octonionic line $\ell_{1}$ (leading coefficient $m=1$ ). Now $\operatorname{Spin}(7)$ acts on the individual octonionic lines $\ell_{0}, \ell_{1}, \ell_{\infty}$, and the only possibility to move planes out of them is through the factor U(1). In fact, the discussion in [3, Chapter 6, p. 44] shows that the factor $\mathrm{U}(1)$ in the group Spin(7)U(1) moves the octonionic lines through the circle, contained in the space $S^{8}$ of the octonionic lines, passing through the three points $m=0,1, \infty$. This corresponds to admitting any real coefficient: $m \in \mathbb{R} \cup \infty$ as slope of the octonionic lines that are admitted to contain the calibrated 4-planes.

Remark 8.2 Following the recent work [15] by J. Kotrbatý, one can use octonionic 1-forms, according to the following formal definitions:

$$
\begin{aligned}
d x & =d \alpha+i d \beta+j d \gamma+k d \delta+e d \epsilon+f d \zeta+g d \eta+h d \theta, \\
\overline{d x} & =d \alpha-i d \beta-j d \gamma-k d \delta-e d \epsilon-f d \zeta-g d \eta-h d \theta, \\
d x^{\prime} & =d \alpha^{\prime}+i d \beta^{\prime}+j d \gamma^{\prime}+k d \delta^{\prime}+e d \epsilon^{\prime}+f d \zeta^{\prime}+g d \eta^{\prime}+h d \theta^{\prime}, \\
\overline{d x^{\prime}} & =d \alpha^{\prime}-i d \beta^{\prime}-j d \gamma^{\prime}-k d \delta^{\prime}-e d \epsilon^{\prime}-f d \zeta^{\prime}-g d \eta^{\prime}-h d \theta^{\prime},
\end{aligned}
$$

referring to pairs of octonions $\left(x, x^{\prime}\right) \in \mathbb{O} \oplus \mathbb{O}=\mathbb{R}^{16}$. Then, in the same spirit proposed in [15], a straightforward computation yields the following formula, much simpler way to write the $\operatorname{Spin}(8)$ canonical 4-form of $\mathbb{R}^{16}$ :

$$
\Phi_{\operatorname{Spin}(8)}=\frac{1}{4}\left(\overline{d x} \wedge d x^{\prime}\right) \wedge\left(\overline{d x^{\prime}} \wedge d x\right) .
$$

Similarly, one gets that the $\operatorname{Spin}(7) \mathrm{U}(1)$ canonical 4-form of $\mathbb{R}^{16}$ can be written in octonionic 1-forms as: 


$$
\begin{aligned}
\Phi_{\mathrm{Spin}(7) \mathrm{U}(1)}= & \frac{1}{4}\left[(\overline{d x} \wedge d x)^{2}+\left(\overline{d x^{\prime}} \wedge d x^{\prime}\right)^{2}\right] \\
& -\frac{1}{2}\left[\left(\overline{d x} \wedge d x^{\prime}\right)^{2}+\left(\overline{d x^{\prime}} \wedge d x\right)^{2}\right]-\left[\left(\overline{d x} \wedge d x^{\prime}\right) \wedge\left(\overline{d x^{\prime}} \wedge d x\right)\right] .
\end{aligned}
$$

Details of both computations are in [3].

Acknowledgements Kai Brynne M. Boydon was supported by University of the Philippines OVPAA Doctoral Fellowship. Part of the present work was done during her visit at Sapienza Università di Roma in the academic year 2018-19, and she thanks Sapienza University and Department of Mathematics "Guido Castelnuovo" for hospitality. Paolo Piccinni was supported by the group GNSAGA of INdAM, by the PRIN Project of MIUR "Real and Complex Manifolds: Topology, Geometry and holomorphic dynamics", and by Sapienza Università di Roma Project "Polynomial identities and combinatorial methods in algebraic and geometric structures".

Funding Open access funding provided by Università degli Studi di Roma La Sapienza within the CRUICARE Agreement.

Open Access This article is licensed under a Creative Commons Attribution 4.0 International License, which permits use, sharing, adaptation, distribution and reproduction in any medium or format, as long as you give appropriate credit to the original author(s) and the source, provide a link to the Creative Commons licence, and indicate if changes were made. The images or other third party material in this article are included in the article's Creative Commons licence, unless indicated otherwise in a credit line to the material. If material is not included in the article's Creative Commons licence and your intended use is not permitted by statutory regulation or exceeds the permitted use, you will need to obtain permission directly from the copyright holder. To view a copy of this licence, visit http://creativecommons.org/licenses/by/4.0/.

\section{References}

1. Alekseevsky, D.V., Chrysikos, I., Fino, A., Raffero, A.: Homogeneous 8-manifolds admitting invariant Spin(7)-structures. Int. J. Math. 31(8), 2050060 (2020)

2. Berger, M.: Du côté de chez Pu. Ann. Sci. École Norm. Sup. 4(5), 1-44 (1972)

3. Boydon, K.B.: Clifford Systems and Clifford Structures with their canonical associated 4-forms in dimensions 8 and 16. Dissertation for the Degree of Doctor of Philosophy in Mathematics, University of the Philippines Diliman (2020)

4. Boyer, Ch. P., Galicki, K.: 3-Sasakian manifolds. Surveys in differential geometry vol. VI: essays on Einstein manifolds, pp. 123-184. Int. Press, Boston, MA (1999)

5. Boyer, C.P., Galicki, K., Mann, B.M., Rees, E.: Compact 3-Sasakian 7-manifolds with arbitrary second Betti number. Invent. Math. 131, 321-344 (1998)

6. Bryant, R.L., Harvey, R.: Submanifolds in hyper-Kähler Geometry. J. Am. Math. Soc. 2(1), 1-31 (1989)

7. Čadek, K., Vanžura, J.: Almost quaternionic structures on eight-manifolds. Osaka J. Math. 35(1), 165190 (1998)

8. Dadok, J., Harvey, R., Morgan, F.: Calibrations on $\mathbb{R}^{8}$. Trans. Am. Math. Soc. 307, 1-40 (1988)

9. Ferus, D., Karcher, H., Münzner, H.F.: Cliffordalgebren und neue isoparametrische Hyperflächen. Math. Z. 177, 479-502 (1981)

10. Harvey, F.R.: Spinors and Calibrations. Academic Press Inc., Cambridge (1990)

11. Harvey, R., Lawson Jr., H.B.: Calibrated geometries. Acta Math. 148, 47-157 (1982)

12. Ivanov, S., Parton, M., Piccinni, P.: Locally conformal parallel $G_{2}$ and $\operatorname{Spin}(7)$ manifolds. Math. Res. Lett. 13(2-3), 167-177 (2006)

13. Joyce, D.D.: Compact Manifolds with Special Holonomy. Oxford University Press, Oxford (2000)

14. Joyce, D.D.: Compact Riemannian manifolds with exceptional holonomy. In: LeBrun, C., Wang, M. (eds.) Surveys in Differential Geometry: Essays on Einstein Manifolds, pp. 39-65. International Press, Vienna (1999)

15. Kotrbatý, J.: Octonion-valued forms and the canonical 8-form on Riemannian manifolds with a Spin(9)-structure. J. Geom. Anal. 30(4), 3616-3640 (2020) 
16. Lotay, J.D.: Calibrated Submanifolds. arXiv:1810.08709v1 (2018)

17. Moroianu, A., Semmelmann, U.: Clifford structures on Riemannian manifolds. Adv. Math. 228, 940967 (2011)

18. Ornea, L., Piccinni, P.: Locally conformal Kähler structures in quaternionic geometry. Trans. Am. Math. Soc. 349(2), 641-655 (1997)

19. Parton, M., Piccinni, P.: Spin(9) and almost complex structures on 16-dimensional manifolds. Ann. Glob. Anal. Geom. 41(3), 321-345 (2012)

20. Parton, M., Piccinni, P.: Parallelizations on products of spheres and octonionic geometry. Complex Manifolds Spec. Issue Complex Geom. Lie Groups 6, 138-149 (2019)

21. Parton, M., Piccinni, P., Vuletescu, V.: Clifford systems in octonionic geometry. Rend. Sem. Mat. Univ. Pol. Torino Workshop Sergio Console 74, 267-288 (2016)

22. Salamon, S.M.: Riemannian Geometry and Holonomy Groups. Longman Sc and Tech., Harlow (1989)

23. Salamon, S.M.: Quaternionic-Kähler geometry. In: LeBrun, C., Wang, M. (eds.) Surveys in Differential Geometry: Essays on Einstein Manifolds, pp. 83-121. International Press, Vienna (1999)

Publisher's Note Springer Nature remains neutral with regard to jurisdictional claims in published maps and institutional affiliations. 